\title{
Steep Eternal Inflation and the Swampland
}

\author{
Konstantinos Dimopoulos* \\ *Consortium for Fundamental Physics, Physics Department, \\ Lancaster University, Lancaster LA1 $4 Y B, U K$ \\ e-mail: k.dimopoulos1@lancaster.ac.uk,
}

\begin{abstract}
I investigate whether eternal inflation is possible when the inflaton originally travels upslope the scalar potential and inevitably reaches a turn-around point where its classical kinetic energy momentarily vanishes. This behaviour occurs regardless of the steepness of the potential slope. Such steep eternal inflation, if achieved, would satisfy the recent swampland conjecture and allow eternal inflation in the string landscape.
\end{abstract}

Eternal inflation is a mode of inflation where the variation of the inflaton field is not determined by its classical motion in field space, but by its quantum fluctuations [1, 2]. Typically, this corresponds to regions of the scalar potential, where the potential slope is shallow and/or the Hubble friction is huge. These regions are called "diffusion zones" because, under the influence of the quantum fluctuations, the inflaton random walks inside the diffusion zone (i.e. its expectation value diffuses in space), assuming equiprobable values throughout the diffusion zone, oblivious of the scalar potential and its slope. Hence, at some locations in space, it is possible for the inflaton to never exit the diffusion zone, in which case inflation never ends. At other locations however, the inflaton exits the diffusion zone and ceases to be dominated by its quantum fluctuations. In the latter case, the inflaton classically rolls down the scalar potential until inflation ends. In this picture, the global universe is undergoing eternal inflation, with the inflaton in the diffusion zone. However, occasionally, in some locations, the inflaton exits the diffusion zone by chance (the quantum fluctuations are random) and classical inflation continues, which eventually ends. Thus, in these locations, we have the formation of a so-called pocket universe, where inflation ends and subsequent evolution ensues. Our Universe is one such pocket universe. So, amids 
eternally inflating space, pocket universes are continuously spawned, with our Universe being one of them.

The original eternal inflation proposal considered a chaotic monomial potential [1], where eternal inflation occurs up the slope near the Planck density, when Hubble friction is huge. In this potential there is only one minimum so all the pocket universes would be identical and would correspond to the locations where the diffused inflaton finds itself at low values and exits the diffusion zone. Soon it was realised that the situation would differ with more complicated potentials, featuring multiple minima. In such a potential the fate of the inflaton, when exiting the diffusion zone, is not unique. Indeed, after eternal inflation the inflaton may find itself in a different minimum at a different location in space. In this case the pocket universes are not identical, but belong to classes corresponding to different minima. This possibility is maximised in the string landscape [3], which features a huge multiple of minima, in the form of valleys and sinks in the multi-field scalar potential of the moduli space [4]. Hence, it has been theorised that most, if not all the minima of the string landscape are populated through eternal inflation, with pocket universes giving rise to many different universes, one of which is our own. This is called the string multiverse. The multiverse idea allowed authors to use anthropic arguments to make sense of a number of tunings apparent in our Universe, that are unexplained but necessary for life to exist [3].

Recently, however, a conjecture was put forward, which requires the slope of the scalar potential in string theory to be bounded from below [5]. In rough terms, it is conjectured that the slope of the potential cannot be shallow, especially on "high ground" where the potential density is large as is the case of primordial inflation. Effective field theories which violate this conjecture cannot be resulting from string theory, so they are not part of the string landscape but instead of the so-called swampland.1 This conjecture may undermine eternal inflation, which seems to require a shallow potential slope. Indeed, the issue was studied in Ref. [10] and it was found that the proposed conjecture does not allow eternal inflation in string theory. As a result, the string landscape cannot be populated and the formation of the string multiverse is questionable.

In this letter, however, we investigate a different mechanism which may

\footnotetext{
${ }^{1}$ For cosmological consequences of this conjecture see Ref. [6, 7]. For critical discussion on this conjecture see Ref. [7, 8]. For discussing Higgs physics in terms of this conjecture see Ref. 9 .
} 
lead to eternal inflation and could operate with steeper potential. The idea is that if the initial conditions of the inflaton send it upwards in the scalar potential, then inevitably, its climb stops at some point, where it turns around and rolls downwards. At the turn-around moment, its classical kinetic density is zero, which means that its quantum fluctuations, no matter how small, may take over. If this were so, then there would be locations in space where the quantum fluctuations of the inflaton conspire to keep it up the scalar potential forever. Can this lead to eternal inflation with a steep potential? If it did, then the formation of the string multiverse would not be threatened by the recent swampland conjecture.

We work in Einstein gravity only. In the following, we use natural units, where $c=\hbar=1$ and Newton's gravitational constant is $8 \pi G=m_{P}^{-2}$, with $m_{P}=2.43 \times 10^{18} \mathrm{GeV}$ being the reduced Planck mass.

First, let us briefly review standard eternal inflation. The idea is as follows. During inflation, gravitational particle production generates perturbations of all light, non-conformally invariant fields. In the case of a light, minimally coupled scalar field, its perturbations are given by the Hawking temperature in de Sitter space $\delta \phi=H / 2 \pi$, which are generated per Hubble time $\delta t=H^{-1}$, where $H$ is the Hubble parameter (approximately constant in inflation). Thus, due to these quantum fluctuations the scalar field is constantly displaced during inflation. This displacement is negligible if it is overwhelmed by the classical variation of the field, as it rolls down the scalar potential. However, if the kinetic density of the classical variation is very small, then the quantum displacement takes over and determines the field evolution. Because quantum fluctuations are random and oblivious of the scalar potential, it is equally possible to push the field towards larger potential values as well as smaller potential values. Thus, if the quantum variation of the field dominates its classical variation and if this field drives the inflationary expansion (the inflaton field) then a fraction of the inflating space will continue inflating forever as the field is kept at high potential values.

The criterion is obtained by comparing the classical to the quantum variation of the field

$$
\frac{\delta \phi}{\delta t}>|\dot{\phi}| \Rightarrow\left|V^{\prime}\right|<\frac{3}{2 \pi} H^{3}
$$

where we also assumed the (classical) slow-roll equation $3 H \dot{\phi} \simeq-V^{\prime}$, where $V$ is the scalar potential density with the dot denoting time derivative and the prime denoting derivative with respect to the scalar field. The above 
suggests that the quantum fluctuations may lead to eternal inflation if the inflaton finds itself at a part of the potential where the potential slope is small enough to satisfy the bound in Eq. (1).

Recently, however, a suggestion was put forward that the effective field theories coming down from string theory (so part of the string landscape) cannot feature a scalar potential with too shallow a slope. Instead it was conjectured that there is a bound on the scalar potential slope of the form

$$
\frac{\left|V^{\prime}\right|}{V}>\frac{c}{m_{P}}
$$

where $c$ is a positive constant of order unity. Using the Friedmann equation in slow-roll $V \simeq 3 H^{2} m_{P}^{2}$ and combining Eqs. (11) and (2) it is straightforward to find that one may feature eternal inflation only when [10]

$$
c<\frac{H}{2 \pi m_{P}} \ll 1
$$

where we consider $H<m_{P}$ to avoid quantum gravity corrections.

Because we see that $c \ll 1$ is needed, it seems that eternal inflation is not possible in the string landscape. Instead, effective field theories which allow eternal inflation find themselves in the swampland. As a result, eternal inflation cannot be used to populate the string landscape and lead to the formation of the string multiverse. Employing the multiverse hypothesis enabled explaining away the cosmological constant problem through anthropic arguments. Without eternal inflation, this seems not as possible.

Can the above situation be remedied? We discuss here a different possibility to realise eternal inflation, even without the requirement of a shallow enough slope in the scalar potential. We call this possibility Steep Eternal Inflation, which if it works, may allow the realisation of the string multiverse without violating the conjecture in Eq. (2).

The basic idea is simple. Suppose that we are in a part of the potential with a steep slope. If the initial conditions are such that the kinetic density of the scalar field is sending the field upwards on the slope 2 then the climbing field reaches a maximum and turns-around rolling down the slope. At the turn-around, the classical kinetic density of the field becomes momentarily zero. Thus, it is likely that near the turn-around the variation of the field

\footnotetext{
${ }^{2}$ There are string theory setups where scalar fields may be forced to climb up the potential after the Big Bang [1].
} 
becomes dominated by its quantum fluctuations. We investigate whether this can lead to eternal inflation, where there are always parts of the Universe where the quantum kicks conspire to keep the field up the slope. Given that, after the exit from spacetime foam, chaotic initial conditions may well lead to the initial kinetic density sending the field upslope, we believe that the the above scenario can be naturally realised.

To model the evolution near the turn-around we Taylor expand the scalar potential near the turn-around point as

$$
V(\phi) \simeq V_{0}+V_{0}^{\prime} \phi
$$

where $V_{0}, V_{0}^{\prime}=$ constant and we have taken the turn-around point to be at $\phi=0$. Based on the above we can take $V^{\prime} / V \simeq V_{0}^{\prime} / V_{0}=$ constant, where we have considered that, because $\phi$ is close to zero, the potential in Eq. (4) is dominated by the constant term $V \simeq V_{0}$. Saturating the inequality in Eq. (2), we can write the constant logarithmic slope as $V^{\prime} / V \simeq c / m_{P}$, where $c>0$ and without loss of generality, we take $V^{\prime}>0$. Thus, the potential, near the turn-around, can be approximated as $V=V_{0} e^{c \phi / m_{P}}$, which in the vicinity of the turn-around point, becomes

$$
V(\phi) \simeq V_{0}\left(1+\frac{c \phi}{m_{P}}\right) .
$$

The above suggests that $V^{\prime} \simeq c V_{0} / m_{P}=$ constant.

Now, let's suppose that the upslope travelling scalar field dominates the Universe. The kinetic density of the field, as it approaches zero, is bound to become smaller than the potential density. Thus, we expect inflation to start (and the field to become the inflaton), and we can write the Friedmann equation as $V \simeq 3 H^{2} m_{P}^{2}$. Now, as we discussed, near the turn-around point, $V \simeq V_{0}=$ constant and so $H \simeq$ constant. The same would be true for the slope of the potential $V^{\prime} \simeq c V / m_{P} \simeq$ constant. Then, the Klein-Gordon equation becomes

$$
\ddot{\phi}+3 H \dot{\phi}+3 c H^{2} m_{P}=0
$$

whose solution is

$$
\dot{\phi}(t)=C e^{-3 H t}-c H m_{P},
$$

where $C \equiv \dot{\phi}(0)+c H m_{P}$ is an integration constant. We see that, only when $\dot{\phi}(0)>0$, i.e. only when the field is originally climbing the upslope of the 
scalar potential, can we reach a turn-around moment $t_{*}$, when $\dot{\phi}\left(t_{*}\right)=0$. From the above, it is straightforward to find that

$$
t_{*}=\frac{1}{3 H} \ln \left(\frac{C}{c H m_{P}}\right) .
$$

As we want to study the system near the turn-around, we Taylor expand the expression in Eq. (77) near $t_{*}$ and find

$$
\dot{\phi}(t)=\dot{\phi}\left(t_{*}\right)+\ddot{\phi}\left(t_{*}\right)\left(t-t_{*}\right)+\cdots \simeq 3 c H^{2} m_{P} \Delta t
$$

where we considered that $\dot{\phi}\left(t_{*}\right)=0, \ddot{\phi}\left(t_{*}\right)=-3 H C e^{-3 H t_{*}}=-3 c H^{2} m_{P}$ and we set $\Delta t=t_{*}-t$, with the ellipsis denoting higher-order terms in $\Delta t$ which we choose to ignore.

To achieve eternal inflation we must satisfy the requirement that

$$
|\dot{\phi}|<\frac{\delta \phi}{\delta t}=\frac{H^{2}}{2 \pi} \Rightarrow \Delta t<\frac{1}{6 \pi c m_{P}} .
$$

The above shows that the total duration when the system satisfies the condition for thermal inflation is $2 \Delta t$, while approaching the turn-around moment and while moving after it. However, in order to have the production of at least one quantum kick, we need that the total duration, while the system satisfies the eternal inflation requirement, is more than a Hubble time, i.e. $2 \Delta t \geq H^{-1}$.This implies the bound

$$
c \leq \frac{H}{3 \pi m_{P}} \ll 1,
$$

which is comparable to the bound in Eq. (3) and demonstrates that in order to achieve eternal inflation in this scenario, one still needs a shallow slope for the scalar potential. If the slope is large, i.e. $c \geq 1$, then the time-interval when the condition of eternal inflation is satisfied (near the turn-around) is much smaller than a Hubble time so there is no time for a single quantum kick 3 Taking into account higher orders in $\Delta t$ in the expansion in Eq. (9) only makes matters worse because enlarging $\dot{\phi}$ makes eternal inflation harder to achieve. Therefore, it seems that the realisation of the string multiverse through eternal inflation is in contradiction with the swampland conjecture in Eq. (2).

\footnotetext{
${ }^{3}$ This means that the quantum displacement of the field is exponentially suppressed.
} 
In summary, we have investigated whether steep eternal inflation is possible and complies with the swampland criterion $\left|V^{\prime} / V\right|>c / m_{P}$ with $c=\mathcal{O}(1)$. To achieve steep eternal inflation we considered a scalar field, initially climbing up its scalar potential until a turnaround point, when momentarily its classical kinetic density becomes zero. We have found that the time period when the quantum variation of the field becomes dominant compared to its classical roll is more than a Hubble time only when $c \ll 1$ and the slope is small enough for the turnaround point to lie in a diffusion zone. This means that steep eternal inflation is not possible and that eternal inflation, under the swampland conjecture, cannot populate the string landscape and realise the string multiverse.

\section{Acknowledgements}

My research is supported (in part) by the Lancaster-Manchester-Sheffield Consortium for Fundamental Physics under STFC grant: ST/L000520/1. I would like to thank $\mathrm{C}$. Owen for igniting my interest in this topic.

\section{References}

[1] A. D. Linde, Mod. Phys. Lett. A 1 (1986) 81.

[2] A. D. Linde, Phys. Lett. B 175 (1986) 395; A. S. Goncharov, A. D. Linde and V. F. Mukhanov, Int. J. Mod. Phys. A 2 (1987) 561; A. H. Guth, J. Phys. A 40 (2007) 6811.

[3] L. Susskind, In *Carr, Bernard (ed.): Universe or multiverse?* 247-266 hep-th/0302219.

[4] N. Arkani-Hamed, L. Motl, A. Nicolis and C. Vafa, JHEP 0706 (2007) 060; H. Ooguri and C. Vafa, Nucl. Phys. B 766 (2007) 21.

[5] G. Obied, H. Ooguri, L. Spodyneiko and C. Vafa, arXiv:1806.08362 [hep-th].

[6] P. Agrawal, G. Obied, P. J. Steinhardt and C. Vafa, Phys. Lett. B 784 (2018) 271; A. Achcarro and G. A. Palma, arXiv:1807.04390 [hep-th]; S. K. Garg and C. Krishnan, arXiv:1807.05193 [hep-th]; A. Kehagias and A. Riotto, arXiv:1807.05445 [hep-th]; M. Dias, 
J. Frazer, A. Retolaza and A. Westphal, arXiv:1807.06579 [hepth]; E. . Colgin, M. H. P. M. Van Putten and H. Yavartanoo, arXiv:1807.07451 [hep-th]; L. Heisenberg, M. Bartelmann, R. Brandenberger and A. Refregier, arXiv:1808.02877 [astro-ph.CO]; W. H. Kinney, S. Vagnozzi and L. Visinelli, arXiv:1808.06424 [astro-ph.CO]; L. Heisenberg, M. Bartelmann, R. Brandenberger and A. Refregier, arXiv:1809.00154 [astro-ph.CO]; C. Damian and O. Loaiza-Brito, arXiv:1808.03397 [hep-th]; M. C. D. Marsh, arXiv:1809.00726 [hepth]; S. Brahma and M. W. Hossain, arXiv:1809.01277 [hep-th]; I. BenDayan, arXiv:1808.01615 [hep-th]; S. Das, arXiv:1809.03962 [hep-th]; D. Wang, arXiv:1809.04854 [astro-ph.CO]; G. D'Amico, N. Kaloper and A. Lawrence, arXiv:1809.05109 [hep-th]; H. Matsui, F. Takahashi and M. Yamada, arXiv:1809.07286 [astro-ph.CO]; M. Motaharfar, V. Kamali and R. O. Ramos, arXiv:1810.02816 [astro-ph.CO]; S. J. Wang, arXiv:1810.06445 [hep-th].

[7] Y. Akrami, R. Kallosh, A. Linde and V. Vardanyan, arXiv:1808.09440 [hep-th].

[8] D. Andriot, arXiv:1806.10999 [hep-th]; C. Roupec and T. Wrase, arXiv:1807.09538 [hep-th]; D. Andriot, arXiv:1807.09698 [hep-th]; J. P. Conlon, arXiv:1808.05040 [hep-th]; H. Murayama, M. Yamazaki and T. T. Yanagida, arXiv:1809.00478 [hep-th].

[9] C. Han, S. Pi and M. Sasaki, arXiv:1809.05507 [hep-ph]; F. Denef, A. Hebecker and T. Wrase, Phys. Rev. D 98 (2018) no.8, 086004; K. Hamaguchi, M. Ibe and T. Moroi, arXiv:1810.02095 [hep-th].

[10] H. Matsui and F. Takahashi, arXiv:1807.11938 [hep-th].

[11] E. Dudas, N. Kitazawa and A. Sagnotti, Phys. Lett. B 694 (2011) 80 